\title{
Regulation of pro-angiogenic tissue factor expression in hypoxia-induced human lung cancer cells
}

\author{
ANDREAS EISENREICH ${ }^{1}$, ANDREAS ZAKRZEWICZ $^{2}$, KILIAN HUBER $^{3}$, HANNES THIERBACH $^{1}$, \\ WOJCIECH PEPKE ${ }^{1}$, PETRA GOLDIN-LANG ${ }^{1}$, HEINZ-PETER SCHULTHEISS ${ }^{1}$, \\ AXEL PRIES $^{2}$ and URSULA RAUCH ${ }^{1}$ \\ ${ }^{1}$ Charité-Universitätsmedizin Berlin, Campus Benjamin Franklin, Centrum für Herz- und Kreislaufmedizin, D-12200 Berlin; \\ ${ }^{2}$ Charité-Universitätsmedizin Berlin, Campus Benjamin Franklin, Institute of Physiology, D-14195 Berlin, Germany; \\ ${ }^{3}$ CeMM Research Center for Molecular Medicine of the Austrian Academy of Sciences, A-1090 Vienna, Austria
}

Received February 4, 2013; Accepted March 20, 2013

DOI: 10.3892/or.2013.2413

\begin{abstract}
Alternative splicing is a key regulatory mechanism for cellular metabolism controlling cell proliferation and angiogenesis, both of which are crucial processes for tumorigenesis under hypoxia. Human cells express two tissue factor (TF) isoforms, alternatively spliced TF (asTF) and 'full length' TF (fITF). flTF is the major source of thrombogenicity whereas, the function of soluble asTF, particularly in cancer, is widely unknown. In the present study, we examined the impact of alternative splicing on the pro-angiogenic potential and the TF expression pattern of A549 cells under hypoxia. We focused our efforts toward alternative splicing factors, such as Clk1, and pro-angiogenic proliferation-regulating factors, such as Cyr61. We further examined the influence of asTF overexpression on the expression of MCP-1, Cyr61 and VEGF, as well as on cell number and pro-angiogenic properties of A549 cells. Notably, we found hypoxia to induce the expression of alternative splicing factors (Clk1 and Clk4) as well as proliferation- and angiogenesis-promoting factors (Cyr61 and flTF). asTF overexpression in A549 cells also increased both cell number and tube formation. These effects were mediated by the induction of Cyr61, MCP-1 and VEGF, as well as by integrin $\alpha_{v} \beta_{3}$. Taken together, our results suggest that the pro-angiogenic potential of A549 lung cancer cells is modulated under hypoxic conditions via modulation of TF isoform expression which in turn is controlled by alternative splicing.
\end{abstract}

Correspondence to: Professor Ursula Rauch or Dr Andreas Eisenreich, Charité-Universitätsmedizin Berlin, Campus Benjamin Franklin, Centrum für Herz- und Kreislaufmedizin, D-12200 Berlin, Germany

E-mail: ursula.rauch@charite.de

E-mail: andreas.eisenreich@charite.de

Key words: tissue factor, splicing, angiogenesis, chemokines, A549

\section{Introduction}

Tissue factor (TF) is known as the primary initiator of the blood coagulation cascade (1-4). Transcription of the TF gene $(F 3)$ leads to the generation of TF pre-messenger RNA (pre-mRNA) consisting of six coding exonic sequences, which are separated by five non-coding introns $(5,6)$. Splicing of this primary transcript results in the removal of all introns, thereby, enabling translation of the remaining six exonic sequences (5). This process yields what is considered to be the mature 'full length' TF (flTF) mRNA $(4,5)$. However, by alternative splicing the 5 th exon may be removed from the TF pre-mRNA, resulting in creation of the mature alternatively spliced TF (asTF) mRNA $(5,7,8)$. Ribosomal translation of both mRNA splice variants finally leads to the generation of two individual TF protein isoforms $(5,6)$. The membranebound flTF was found to be essential for the thrombogenicity of cells and tissues $(2,9)$. In contrast, the function of asTF is not well studied. Although asTF is able to promote FXa generation in the presence of phospholipids, its pro-coagulant activity is much lower than that of flTF $(4,5)$. Some studies have suggested that asTF may be linked to increased tissue growth and angiogenesis (10-13).

Cancer is a leading cause of hospitalization and death in the world (14). Both angiogenesis and cell proliferation, which are important for tumorigenesis and growth, have been shown to be promoted by hypoxic conditions. Other factors stimulating these processes are monocyte chemotactic protein-1 (MCP-1, CCL-2), cysteine-rich 61 (Cyr61, CCN1) or vascular endothelial growth factor (VEGF) (15-17). Integrin interaction with these factors is often involved in pro-angiogenic and proliferation-facilitating processes and has been associated with the pathogenesis of cancer in general (18-20). Several studies have demonstrated that the expression of asTF is significantly increased in a variety of cancer cells $(3,14,21)$. Moreover, elevated asTF levels were found to be associated with increased angiogenesis in vivo and to accelerate tumor growth in mice (12). Recently, van den Berg et al (13) reported asTF to boost angiogenesis via integrin signaling in endothelial cells. Yet, it remains unclear 
how asTF induces integrin-mediated angiogenesis and tumor growth.

In the present study, we set out to characterize the impact of post-transcriptional splicing regulation by $\mathrm{Cdc} 2$-like kinases (Clks) on the modulation of pro-angiogenic properties of A549 lung cancer cells under hypoxic conditions. In this context, we investigated the effect of stable asTF overexpression on expression levels of pro-angiogenic and proliferation-promoting factors, such as MCP-1, Cyr61 and VEGF in A549 cells. We also determined the impact of asTF on cell number and angiogenesis.

Overall, our results suggest that post-transcriptional splicing regulation is able to modulate the pro-angiogenic potential of A549 cancer cells. Furthermore, the data imply that this effect is, at least in part, caused by differential isoform expression of TF and induction of proliferation-facilitating pro-angiogenic factors, such as MCP-1, Cyr61 and VEGF, as well as integrin-mediated signaling.

\section{Materials and methods}

Cell culture. Human lung carcinoma cells (A549) (PromoCell $\mathrm{GmbH}$, Heidelberg, Germany) were cultured in RPMI medium [containing 10\% fetal calf serum (FCS); PAA Laboratories $\mathrm{GmbH}$, Pasching, Austria] at $37^{\circ} \mathrm{C}$ in a humidified incubator (5\% $\mathrm{CO}_{2}, 95 \%$ air). Human microvascular endothelial cells (HMEC-1) (PromoCell GmbH) were cultured in endothelial cell growth medium (containing 5\% FCS; PromoCell GmbH). For antibody-mediated inhibition, A549 cells or cell supernatant were incubated with $5 \mu \mathrm{g} / \mathrm{ml}$ of specific neutralizing antibodies against Cyr61 (a kind gift from Lester F. Lau, University of Illinois, Chicago, IL, USA); MCP-1 and VEGF were provided by R\&D Systems (Minneapolis, MN, USA), and flTF (\#4501) was from American Diagnostica Inc. (Greenwich, CT, USA). Compound-based experiments were performed using the $\alpha_{\mathrm{v}} \beta_{3}$ inhibitor cyclic RGD (cRGD, H-4772 from Bachem AG, Bubendorf, Switzerland; $10-\mu \mathrm{M}$ final concentration) or the Clk1- and Clk4-specific inhibitor KH-CB19 (a generous gift from Professor Franz Bracher, Department of Pharmacy, Center for Drug Research, Ludwig Maximilians University of Munich, Munich, Germany) (22). Controls were stimulated with $100 \mathrm{nM}$ recombinant human MCP-1 (R\&D Systems) or $50 \mu \mathrm{g} / \mathrm{ml}$ recombinant human asTF (a kind gift from Vladimir Y. Bogdanov, University of Cincinnati College of Medicine, Cincinnati, OH, USA); negative controls were not treated. Hypoxia was induced by incubation of cells at $37^{\circ} \mathrm{C}$ in a humidified incubator $\left(5 \% \mathrm{CO}_{2}, 95 \%\right.$ air $)$ in a reduced $\mathrm{O}_{2}$ environment $(3 \%)$. siRNA-mediated inhibition was conducted by transfection with Clk1- or Clk4-specific siRNAs (100 pmol) or nonsense control siRNA (100 pmol; Applied Biosystems, Darmstadt, Germany). Transfection was performed using Lipofectamine ${ }^{\mathrm{TM}} 2000$ (Invitrogen $\mathrm{GmbH}$, Karlsruhe, Germany). For expression analyses (mRNA and protein level), time courses from 0 to $24 \mathrm{~h}$ were performed in preliminary tests to assess the optimal time points (data not shown). These time points were then used for expression analyses as indicated in the results.

Overexpression plasmids. For transfection experiments, a cDNA construct of asTF was cloned into the multiple cloning site of the pVITRO2 vector (InvivoGen, San Diego, CA, USA). The quality, orientation and identity of the overexpression vector and the insert were confirmed by automated sequencing.

Stable transfection of A549 cells. A594 cells (5x10\%/well) were seeded in 6-well plates. Transfection of pVITRO2_asTF or pVITRO2_LV was conducted using Lipofectamine ${ }^{\mathrm{TM}} 2000$ following the manufacturer's protocol. Selection of stable transfectants was performed by incubating transfected cells with $250 \mathrm{ng} / \mathrm{ml}$ hygromycin (PAA Laboratories $\mathrm{GmbH}$ ). Stable overexpression was continuously verified by real-time PCR.

Semi-quantitative RT-PCR. Total RNA was reverse transcribed using AMV (Roche Diagnostics GmbH, Mannheim, Germany), and cDNAs encoding MCP-1, Cyr61 and VEGF, were amplified. Primers used for detection were: MCP-1 forward, 5'-TCTGTGCCTGCTGCTCATAG-3' and MCP-1 reverse, 5'-CAGATCTCCTTGGCCACAAT-3'; Cyr61 forward, 5'-TCCTCTGTGTCCCCAAGAAC-3' and Cyr61 reverse, 5'-TTCAGGCTGCTGTACACTGG-3'; VEGF forward, 5'-TCC AGGAGTACCCTGATGAGA-3' and VEGF reverse, 5'-GCT TGTCACATCTGCAAGTACG-3' (Ocimum Biosolutions Ltd., Hyderabad, India). GAPDH-, Clk1-, Clk-4-, flTF- and asTF-specific primers were as previously described (4). RT-PCR conditions consisted of $94^{\circ} \mathrm{C}$ for $2 \mathrm{~min}$, and 36 cycles of $94^{\circ} \mathrm{C}$ for $30 \mathrm{sec} ; 60^{\circ} \mathrm{C}$ for $25 \mathrm{sec}$; and $72^{\circ} \mathrm{C}$ for $1 \mathrm{~min}$. Additionally, HIF-1 $\alpha$ mRNA expression was determined as control for the induction of the hypoxia response. Primers used for detection were: HIF-1 $\alpha$ forward, 5'-CACCTCTGGACT GCCTTTC-3' and HIF-1 $\alpha$ reverse, 5'-GAAGTGGCAACT GATGAGCA-3'. RT-PCR conditions for HIF-1 $\alpha$ consisted of $94^{\circ} \mathrm{C}$ for $2 \mathrm{~min}$, and 36 cycles of $94^{\circ} \mathrm{C}$ for $30 \mathrm{sec} ; 58^{\circ} \mathrm{C}$ for $25 \mathrm{sec}$; and $72^{\circ} \mathrm{C}$ for $30 \mathrm{sec}$. The impact of $\mathrm{Clk}$ inhibition on TF splicing was assessed at the mRNA level following the protocol by Schwertz et al (23). Post-transcriptional processing of unspliced human TF pre-mRNA (pTF) to spliced mature human TF mRNA (mTF) was measured using the specific primers: TF-exon 4 forward, 5'-CTCGGACAGCCA ACAATTCAG-3' and TF-exon 5 reverse, 5'-CGGGCTGTCT GTACTCTTCC-3' (23). The corresponding RT-PCR product pTF (904 bp) includes a part of exon 4, intron 4, and a part of exon 5 . In contrast to pTF, the mTF amplicon (297 bp) only includes exon 4 and exon 5 but not the intronic sequence due to the exclusion of this intron via post-transcriptional splicing. Since the expression level of pTF is much lower than that of mTF, the cycle numbers for the RT-PCRs were adjusted. RT-PCR conditions for pTF (and mTF) consisted of $94^{\circ} \mathrm{C}$ for $2 \mathrm{~min}$, and 40 cycles $\left(27\right.$ cycles for mTF) of $94^{\circ} \mathrm{C}$ for $30 \mathrm{sec}$; $60^{\circ} \mathrm{C}$ for $25 \mathrm{sec}$; and $72^{\circ} \mathrm{C}$ for $1 \mathrm{~min}$.

Tube formation assay. To analyze angiogenesis, the In Vitro Angiogenesis Assay kit from Millipore (Billerica, MA, USA) was used. The assay was performed following the manufacturer's protocol. In brief, HMEC-1 cells $\left(7.5 \times 10^{3} /\right.$ well) were resuspended in the supernatant of asTF-overexpressing A549 cells or cells transfected with a control plasmid (LV). Fresh RPMI containing $100 \mathrm{nM}$ recombinant MCP-1 or $50 \mu \mathrm{g} / \mathrm{ml}$ recombinant asTF was used as positive controls. The impact of hypoxia was determined using the supernatant of A549 
cells incubated for $24 \mathrm{~h}$ under hypoxic $\left(3 \% \mathrm{O}_{2}\right)$ or normoxic conditions $\left(20 \% \mathrm{O}_{2}\right)$, identical to the conditions used for protein expression analyses via western blotting. Subsequently, HMEC-1 cells were seeded on $50 \mu 1$ of the ECMatrix ${ }^{\mathrm{TM}}$ in 96-well plates in $150 \mu 1$ of the A549 supernatants as mentioned above and incubated for $7 \mathrm{~h}$ at $37^{\circ} \mathrm{C}$ in a humidified air incubator with $5 \% \mathrm{CO}_{2}$. This time point was found to be optimal for the measurement of tube formation by HMEC-1 cells in this experimental setting. After $7 \mathrm{~h}$, the number of rings formed by endothelial cells was counted and compared to the corresponding controls. Tube formation by endothelial cells was analyzed using a Leica DMIL light microscope (Leica, Wetzlar, Germany).

MTT assay. Cell proliferation of A549 cells was determined by MTT assay. This colorimetric assay determines the activity of cellular enzymes that reduce the yellow tetrazolium salt (3-(4,5-dimethylthiazol-2-yl)-2,5-diphenyltetrazolium bromide (MTT) to a purple formazan dye in living cells. The MTT assay measures the cell proliferation via determination of the cellular metabolic activity of $\mathrm{NAD}(\mathrm{P}) \mathrm{H}$-dependent oxidoreductase enzymes. Here, $2.5 \times 10^{4}$ cells in $100 \mu$ l RPMI medium/well were seeded in 96-well plates. Cells were incubated for $48 \mathrm{~h}$ with or without inhibitory antibodies or cRGD at $37^{\circ} \mathrm{C}$ in humidified air with $5 \% \mathrm{CO}_{2}$. After the incubation, $25 \mu 1$ of MTT $(5 \mathrm{mg} / \mathrm{ml})$ was added to each well and incubated at $37^{\circ} \mathrm{C}$ for $3 \mathrm{~h}$. The reaction was stopped by adding solubilization solution. The plates were further incubated for $1 \mathrm{~h}$ at $37^{\circ} \mathrm{C}$. Subsequently, the concentration of the generated formazan was determined by UV absorption at $570 \mathrm{~nm}$ in a VersaMax Microplate Reader (Molecular Devices GmbH, Ismaning, Germany). Cell number was determined using a standard curve from 0 to 10,000 cells/100 $\mu 1$ of media and well.

Western blotting. Western blot analysis of samples from the A549 cell lysates was performed as previously described (4). For analysis of asTF in the supernatant of A549 cells, the medium was collected after $24 \mathrm{~h}$, respectively. Soluble proteins in the supernatant were precipitated by treatment with trichloroacetic acid (TCA) overnight at $4^{\circ} \mathrm{C}$. Since there is no established protein loading control for secreted proteins, equal protein loading was confirmed by determining the amount of total protein via a BCA assay. For western blot experiments, $20 \mu \mathrm{g}$ of whole protein was used. For detection, specific antibodies against fITF, asTF and GAPDH as previously described (4), MCP-1 (Santa Cruz Biotechnology, Santa Cruz, CA, USA) VEGF (Abnova GmbH, Heidelberg, Germany), Clk1 and Clk4 (Aviva Systems Biology, San Diego, CA, USA) and Cyr61 (a kind gift from Lester F. Lau, University of Illinois, Chicago, IL, USA) were used.

Quantification of western blots and RT-PCR. The results of western blot and RT-PCR experiments were quantified using Gel-Pro Analyzer ${ }^{\mathrm{TM}}$ software version 4.0.00.001 (Media Cybernetics, Bethesda, MD, USA).

TF isoform-specific real-time PCR (TaqMan). Real-time PCR employing fITF-, asTF- and GAPDH-specific primers and probes was performed as previously described (4). Quantification of real-time PCR data was performed by
A
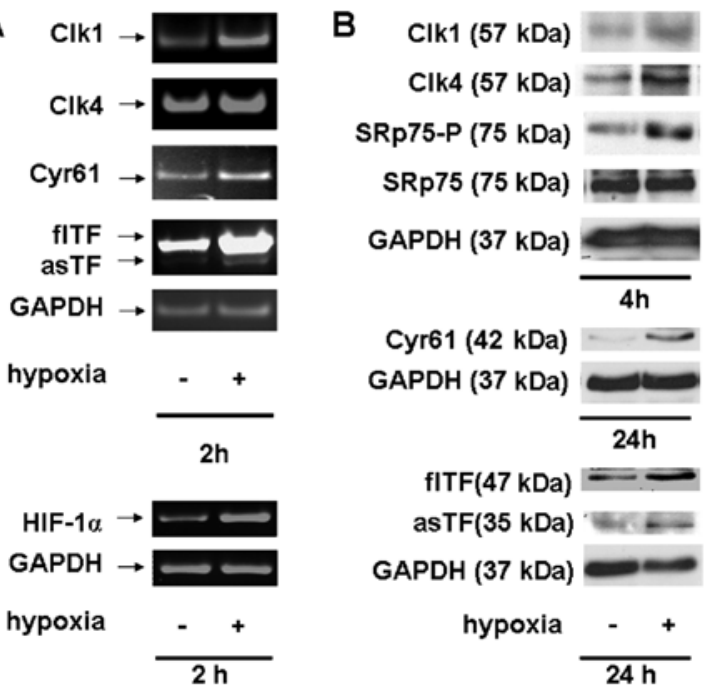

$4 \mathrm{~h}$

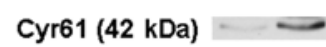

GAPDH (37 kDa)

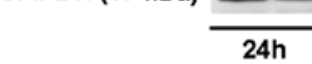

$\mathrm{fITF}(47 \mathrm{kDa})=$

asTF(35 kDa)

GAPDH $(37 \mathrm{kDa})$

hypoxia - +

$2 \mathrm{~h}$

$24 \mathrm{~h}$

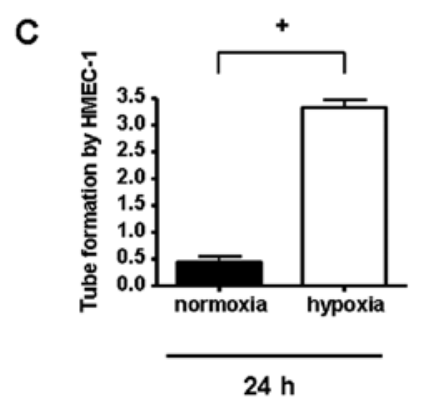

Figure 1. Hypoxia alters the expression pattern and the pro-angiogenic potential of A549 cells. (A) mRNA expression of Clk1 (347 bp), Clk4 (440 bp), Cyr61 (450 bp), flTF (931 bp), asTF (771 bp), HIF-1 $\alpha$ (252 bp) and GAPDH (185 bp) in A549 cells $2 \mathrm{~h}$ post hypoxia induction; $\mathrm{n} \geq 3$. (B) Western blot analysis of intracellular expression of Clk1 (57 kDa), Clk4 (57 kDa), phosphorylated SRp75 (75 kDa), Cyr61 (42 kDa), fTTF (47 kDa), asTF (35 kDa) and GAPDH (37 kDa) in A549 cells 4 or $24 \mathrm{~h}$, respectively, after induction of hypoxic conditions; $n \geq 3$. (C) Tube formation assay of HMEC-1 cells treated with the supernatant of hypoxia-treated A549 cells post $5 \mathrm{~h}$. ${ }^{+} \mathrm{P}<0.01, \mathrm{n} \geq 3$.

employing standard curves of double-stranded cDNA fragments covering the complete coding sequence for each target (asTF, fITF and GAPDH). Results of real-time PCRs for fITF and asTF were normalized against GAPDH. Statistical analyses were carried out using GraphPad Prism 4 version 4.03 (GraphPad Software Inc., La Jolla, CA, USA).

Measurement of TF activity. Determination of total pro-coagulant activity of A549 cells was performed as previously described (4).

Statistical analysis. All data are expressed as means \pm SEM. Data were analyzed by the Student's t-test or one-way ANOVA. A probability value $\leq 0.05$ was considered to indicate a statistically significant result.

\section{Results}

Hypoxia induces a modulated TF expression pattern in A549 cells. To determine the impact of hypoxia on TF isoform expression and other angiogenic factors, A549 cells were incubated under hypoxic conditions $\left(3 \% \mathrm{O}_{2}\right)$ for 2,4 or $24 \mathrm{~h}$, respectively. Compared to cells cultured under normoxic conditions, 
A
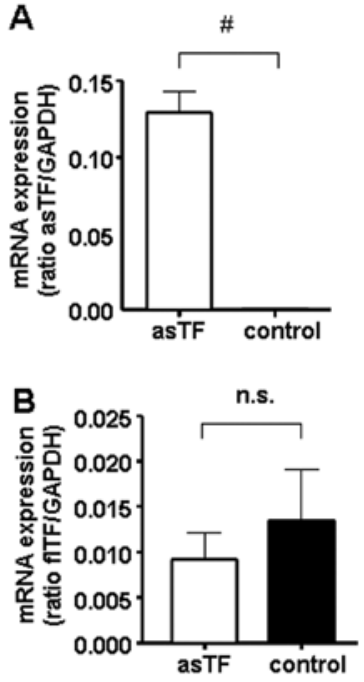

C
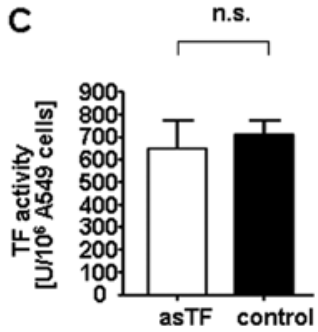

Figure 2. Stable overexpression of asTF in A549 cells has no impact on cellular thrombogenicity. (A) asTF mRNA expression in A549 cells stably transfected with asTF or the empty control plasmid (control); $n \geq 4$. (B) fITF mRNA expression, $\mathrm{n} \geq 4$. Ratio of the TF mRNA expression normalized to GAPDH of $5 \times 10^{5}$ A5 49 cells. Means \pm SEM are shown. (C) TF activity of asTF-overexpressing A549 cells compared to mock-transfected controls. Data are displayed as arbitrary units of TF activity per $1 \times 10^{6}$ A549 cells. The mean \pm SEM of 7 independent experiments is shown $(n \geq 7)$. ${ }^{\#} \mathrm{P}<0.001$; and n.s., no significant difference.

hypoxia increased the mRNA expression of both TF isoforms, flTF and asTF, as well as the pro-angiogenic mediators Cyr61, HIF-1 $\alpha$, and other factors involved in alternative splicing, such as Clk1 and Clk4 (Fig. 1A). Consistent with these data, protein levels of TF isoforms and Cyr61 were also found to be increased under hypoxic conditions (Fig. 1B). Moreover, this setting also led to a significant increase in the protein expression of the alternative splicing-modulating kinases, Clk1 and $\mathrm{Clk} 4$, and strongly affected the phosphorylation of the known Clk downstream effector SRp75. Furthermore, treatment of microvascular endothelial cells (HMEC-1) with the supernatant of A549 cells incubated for $24 \mathrm{~h}$ under hypoxic conditions induced pro-angiogenic tube formation of HMEC-1 in vitro (Fig. 1C). Together these data indicate that hypoxia triggers the switch from a normal to a pro-angiogenic state.

Effect of asTF overexpression on $m R N A$ and protein levels in A549 cells. To assess whether asTF mediates pro-angiogenic effects, asTF was stably overexpressed in A549 cells followed by evaluation of the expression of pro-angiogenic factors as well as cell number and angiogenesis induction. Compared to cells transfected with the empty control plasmid (LV), stable asTF overexpression (asTF) was associated with a significant increase in asTF, but not flTF, at the mRNA level (Fig. 2A
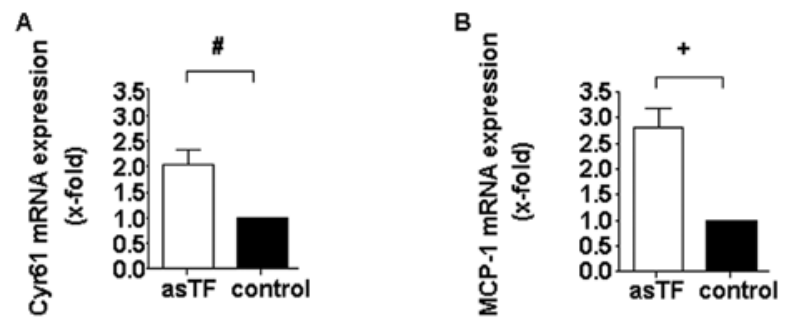

\section{C}

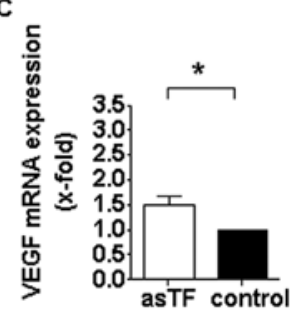

D

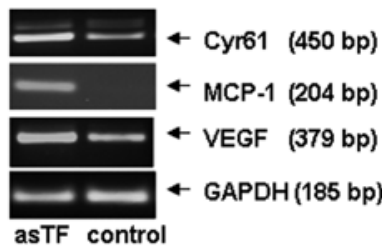

E
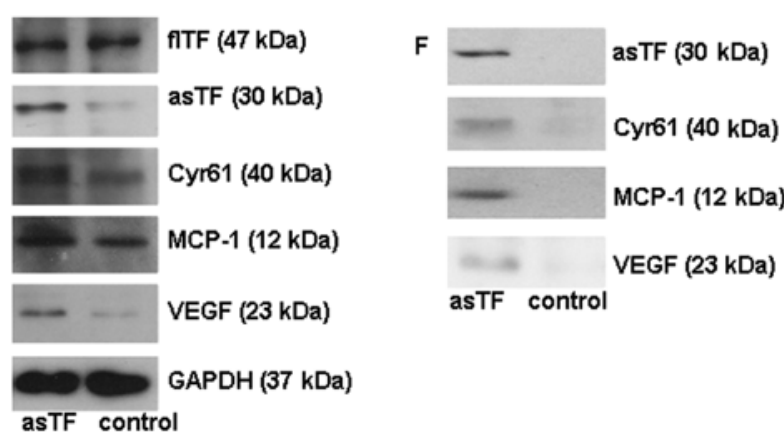

Figure 3. Stable overexpression of asTF modifies the expression pattern of A549 cells. Shown is the quantification of the mRNA expression of (A) Cyr61 (450 bp), (B) MCP-1 (204 bp) and (C) VEGF (379 bp) in asTF-overexpressing A549 cells (asTF) compared to mock-transfected controls (control). (A-C) The quantification of the mRNA expression is displayed as the OD of band density and is expressed as the means \pm SEM. ${ }^{*} \mathrm{P}<0.05 ;{ }^{+} \mathrm{P}<0.01 ;{ }^{\#} \mathrm{P}<0.001$; and n.s., no significant difference; $n \geq 3$. (D) Representative RT-PCR gels. $\mathrm{n} \geq 3$. GAPDH (185 bp) was used as loading control. (E) Western blot analysis of intracellular expression of flTF (47 kDa), asTF (30 kDa), Cyr61 (40 kDa), MCP-1 (12 kDa), VEGF (23 kDa) and GAPDH (37 kDa) in transfected or nontransfected A549 cells pre-treated with or without $10 \mu \mathrm{M}$ of $\mathrm{KH}-\mathrm{CB} 19$ and incubated for $24 \mathrm{~h}$ under hypoxic conditions; $\mathrm{n} \geq 3$. (F) Detection of secreted asTF, Cyr61, MCP-1 and VEGF in the supernatant of asTF-overexpressing A549 cells compared to the corresponding controls; $n \geq 3$.

and B). Overall, expression levels of asTF and flTF mRNA were comparable in asTF-overexpressing cells. Stable asTF overexpression also significantly induced the expression of the pro-angiogenic factors Cyr61, MCP-1 and VEGF (Fig. 3A-E). In addition, the total amount of asTF and the other abovementioned pro-angiogenic factors secreted into the supernatant was increased compared to cells transfected with the control plasmid (Fig. 3F).

Overexpression of the soluble TF isoform had no influence on cellular thrombogenicity. AsTF is assumed to exhibit a low pro-thrombogenic potential yet flTF appears to be the major contributor to thrombogenicity. To confirm these data 
A
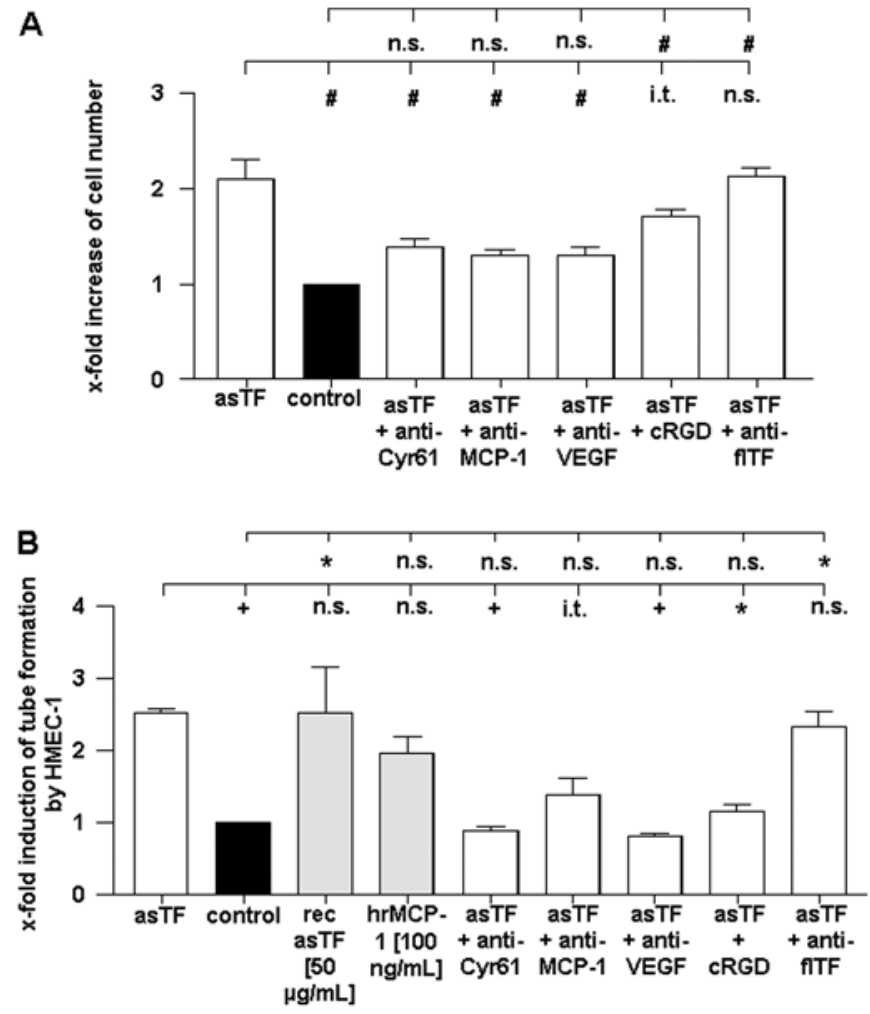

Figure 4. AsTF increases A549 cell number and facilitates pro-angiogenic tube formation by HMEC-1 cells. (A) Results of the MTT cell proliferation assay. Shown is the increase in the cell number of asTF-overexpressing cells compared to cells stably transfected with an empty control plasmid (control). The impact of antibody-mediated or pharmacologic inhibition of Cyr61 (asTF + anti-Cyr61), MCP-1 (asTF + anti-MCP-1), VEGF (asTF + anti-VEGF), flTF (asTF + anti-flTF) and $\alpha_{v} \beta_{3}($ asTF + cRGD) was characterized. Means \pm SEM are shown; $n \geq 10$. (B) Depicted is the $x$-fold induction of tube formation by HMEC-1 treated with the supernatant of asTF-overexpressing cells and cells stably transfected with an empty control plasmid (control). Endothelial cells stimulated with $50 \mu \mathrm{g} / \mathrm{ml}$ recombinant asTF (rec asTF) or $100 \mathrm{ng} / \mathrm{ml}$ human recombinant MCP-1 (hrMCP-1), respectively, were used as positive controls. The impact of antibody-mediated or pharmacologic inhibition of Cyr61 (asTF + anti-Cyr61), MCP-1 (asTF + anti-MCP-1), VEGF (asTF + anti-VEGF), flTF (asTF + anti-fTF) and $\alpha_{v} \beta_{3}$ (asTF + cRGD) was characterized in the supernatant of asTF-overexpressing A549 cells. Means \pm SEM are depicted; $\mathrm{n} \geq 6 .{ }^{*} \mathrm{P}<0.05 ;{ }^{+} \mathrm{P}<0.01 ;{ }^{\#} \mathrm{P}<0.001$; ${ }^{\text {i.t. }} \mathrm{P}<0.15$ (in trend); n.s., no significant difference.

in our experimental setting, the impact of asTF overexpression on the pro-coagulant activity of A549 cells was analyzed using a chromogenic TF activity assay. As shown in Fig. 2C, TF activity of A549 cells was not significantly influenced by stable asTF overexpression in comparison to the corresponding controls (Fig. 2C).

Impact of asTF overexpression on cell number. Overexpression of asTF increased the number of A549 cells by 2 -fold compared to cells transfected with the empty control vector (Fig. 4A). However, inhibition of the pro-angiogenic factors Cyr61, MCP-1, as well as VEGF by specific neutralizing antibodies significantly reduced the pro-proliferative effect mediated by asTF. Moreover, pharmacologic inhibition of integrin $\alpha_{v} \beta_{3}$ also led to a significant reduction in the number of asTF-overexpressing cells. In contrast to the inhibition of integrin $\alpha_{v} \beta_{3}$ and the above mentioned pro-angiogenic factors, the neutralization of flTF by specific inhibitory antibodies had no influence on cell number in the asTF-overexpressing A549 cells (Fig. 4A).

Supernatant of asTF-overexpressing A549 cells induces endothelial tube formation. To characterize the pro-angiogenic potential of asTF overexpression, we investigated the effect of the cell supernatant on in vitro tube formation of human endothelial cells (HMEC-1) (Fig. 4B). To elucidate the individual effects of different components within the supernatant, we used neutralizing antibodies and a pharmacologic integrin $\alpha_{v} \beta_{3}$ inhibitor. MCP-1 as well as recombinant asTF were applied as positive controls for the induction of tube formation by HMEC-1.

As shown in Fig. 4B, the supernatant of asTF-overexpressing A549 cells increased the tube formation by HMEC-1 compared to the supernatant of cells transfected with a control plasmid. The degree of the pro-angiogenic potential of the asTF-containing supernatant was comparable to that of recombinant asTF or MCP-1 on endothelial tube formation (Fig. 4B). Antibody-mediated depletion of Cyr61 and VEGF significantly reduced the pro-angiogenic effect of the supernatant of asTFoverexpressing A549 cells. Similarly, blocking of MCP-1 led to a slight decrease in tube formation. Pharmacologic inhibition of integrin $\alpha_{v} \beta_{3}$ also reduced the effect of the asTF-containing supernatant of HMEC-1 in this assay. In contrast, inhibition of fITF by neutralizing antibodies had no effect on the increase in endothelial tube formation (Fig. 4B).

Together, these data indicate that asTF induces proliferation as well as the pro-angiogenic potential of A549 cells without affecting the pro-thrombogenicity of these cells. Based on these results, we conclude that asTF mediates its effects via several proliferation-modulating and pro-angiogenic factors including Cyr61 and MCP-1, as well as via integrin $\alpha_{v} \beta_{3}$ signaling.

Clks modulate the TF isoform expression under hypoxic conditions. Next, we aimed to ascertain how TF isoform expression is regulated at the post-transcriptional level by alternative splicing when hypoxic conditions are applied.

As expected, we found an induction of flTF and asTF mRNA expression in A549 cells when comparing hypoxic vs. normoxic conditions (Fig. 5A and B). Treatment of cells with the Clk1- and Clk4-specific inhibitor KH-CB19 (22) led to a significant reduction in both fTF and asTF independent of the amount of oxygen provided during incubation. To verify these data, we transfected A549 cells with specific siRNAs targeting Clk1 and Clk4. Consistent with the results obtained with KH-CB19, treatment of cells with siRNA either against $\mathrm{Clk} 1$ and $\mathrm{Clk} 4$ alone or in combination significantly reduced mRNA expression of fITF and asTF under normoxic as well as under hypoxic conditions (Fig. 5C and D).

To determine whether pharmacologic inhibition of Clk1 and Clk4 also influences the protein expression of the TF isoforms, we performed western blot analyses. In agreement with the previous mRNA data, hypoxia induced protein expression of both TF isoforms after $24 \mathrm{~h}$ (Fig. 5E and F). Treatment with the Clk1 and Clk4 inhibitor (Fig. 5E) or siRNAs against $\mathrm{Clk} 1$ and $\mathrm{Clk} 4$ (Fig. 5F) reduced protein expression of both TF isoforms in both high and low oxygen setting. 
A

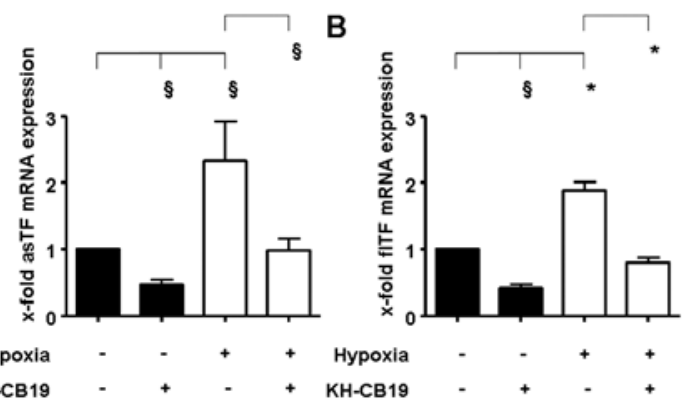

E

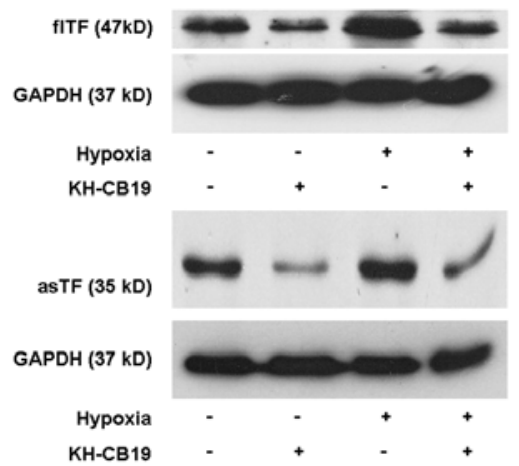

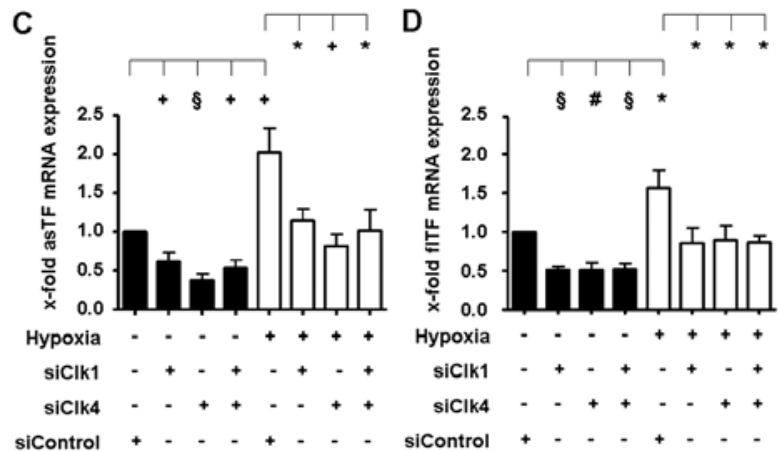

$\mathbf{F}$

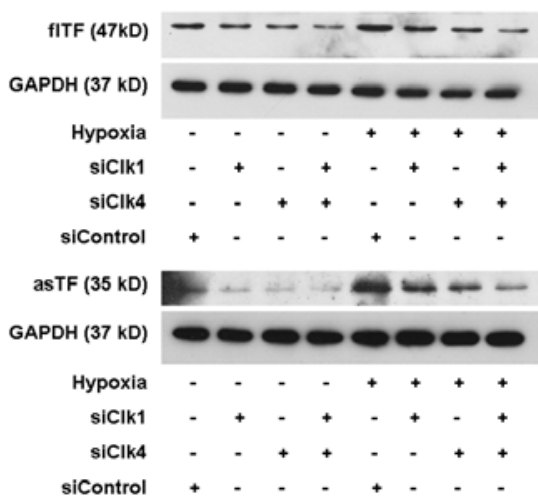

Figure 5. Clk1 and Clk4 modulate the TF isoform expression under hypoxic and normoxic conditions. (A-D) Ratio of the TF mRNA expression normalized against GAPDH of $5 \times 10^{5}$ A549 cells $2 \mathrm{~h}$ post hypoxia induction. (A) asTF or (B) flTF mRNA expression of A549 cells pre-treated with or without the pharmacologic $\mathrm{Clk} 1$ and $\mathrm{Clk} 4$ inhibitor $(\mathrm{KH}-\mathrm{CB} 19 ; 10 \mu \mathrm{M})$, and incubated for $2 \mathrm{~h}$ under hypoxic conditions compared to the corresponding controls treated under normoxic conditions; $\mathrm{n} \geq 7$. Isoform mRNA expression of (C) asTF and (D) fITF $48 \mathrm{~h}$ post transfection in 1x10 ${ }^{6}$ A549 cells transfected with 100 pmol siRNAs against Clk1 (siClk1), Clk4 (siClk4), or a scrambled nonsense control siRNA (siControl), respectively, and $2 \mathrm{~h}$ after hypoxic induction; $\mathrm{n} \geq 5$. Means \pm SEM are shown. ${ }^{\mathrm{P}}<0.05 ;{ }^{+} \mathrm{P}<0.01 ;{ }^{*} \mathrm{P}<0.001 ;{ }^{\circledR} \mathrm{P}<0.0001$; n.s., no significant difference. (E) Western blot analyses of the intracellular protein expression of flTF $(47 \mathrm{kDa})$ and asTF $(35 \mathrm{kDa})$ in A549 cells pre-treated with or without $10 \mu \mathrm{M}$ of KH-CB19 and incubated for $24 \mathrm{~h}$ under hypoxic conditions. GAPDH (37 kDa) was used as a loading control; $n \geq 3$. (F) Western blot analysis of the protein expression of flTF and asTF in A549 cells transfected with specific siRNAs against Clk1 and Clk4 for $48 \mathrm{~h}$ and incubated under hypoxic conditions for $24 \mathrm{~h}$. The corresponding controls were incubated under normoxia; $\mathrm{n} \geq 3$.

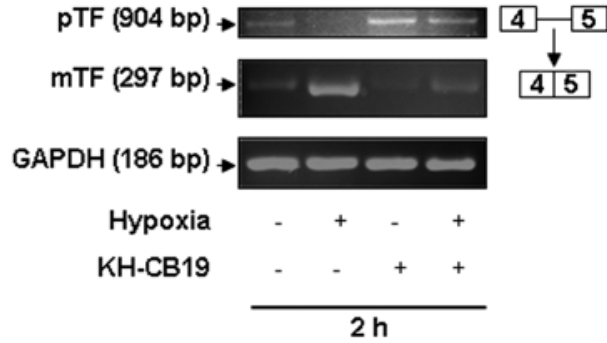

Figure 6. Impact of Clk inhibition on TF splicing in A549 cells. Shown are the results of semi-quantitative RT-PCR analyses of the splicing of human TF pre-mRNA (pTF) to mature TF mRNA (mTF) under normoxic as well as hypoxic conditions. The non-spliced pTF form (904 bp) contains intron 4 (black line) which is located between exon 4 (box including the number '4') and exon 5 (box including the number ' 5 '). The exclusion of intron 4 via mRNA splicing led to the formation of the smaller mature mTF form (297 bp). Compared are A549 cells pre-treated with or without the pharmacologic Clk inhibitor (KH-CB19; $10 \mu \mathrm{M})$. The cells were incubated $2 \mathrm{~h}$ under hypoxic or normoxic conditions, respectively. GAPDH (185 bp) was used as a loading control; $\mathrm{n} \geq 4$.

In order to further validate the impact of Clks on TF splicing, we also investigated the post-transcriptional processing of unspliced human TF pre-mRNA (pTF) to spliced mature human TF mRNA (mTF) by semi-quantitative RT-PCR as described by Schwertz et al (Fig. 6) (23). Under normoxic conditions both pTF as well as mTF were detected at the mRNA level in A549 cells (Fig. 6). As shown, hypoxia increased the amount of the spliced mTF form and reduced the pTF level. Treatment of cells with the specific Clk inhibitor $\mathrm{KH}-\mathrm{CB} 19(10 \mu \mathrm{M})$ led to a significant reduction in the spliced mTF. In contrast, the level of the unspliced pTF form was increased in these cells. The same results were observed both under normoxic as well as under hypoxic conditions.

Impact of Clk inhibition on the pro-angiogenic potential of A549 cells. We performed HMEC-1 tube formation assays to elucidate the impact of Clk inhibition on the pro-angiogenic potential of A549 cells. HMEC-1 cells treated with the supernatant of A549 cells which had been incubated for $24 \mathrm{~h}$ under hypoxic conditions displayed an induction in tube formation (Fig. 7). Inhibition of Clk1 and Clk4 by KH-CB19 as well as treatment with the siRNA targeted against Clk1 and Clk4 significantly reduced the hypoxia-induced tube formation by HMEC-1 cells, whereas nonsense siRNA controls had no influence on this effect. The low pro-angiogenic potential of the supernatant of A549 cells incubated under normoxic conditions was not altered by Clk inhibition (Fig. 7).

In summary, these data indicate that Clks play an important role in the regulation of the pro-angiogenic properties of A549 cells under hypoxic conditions. 


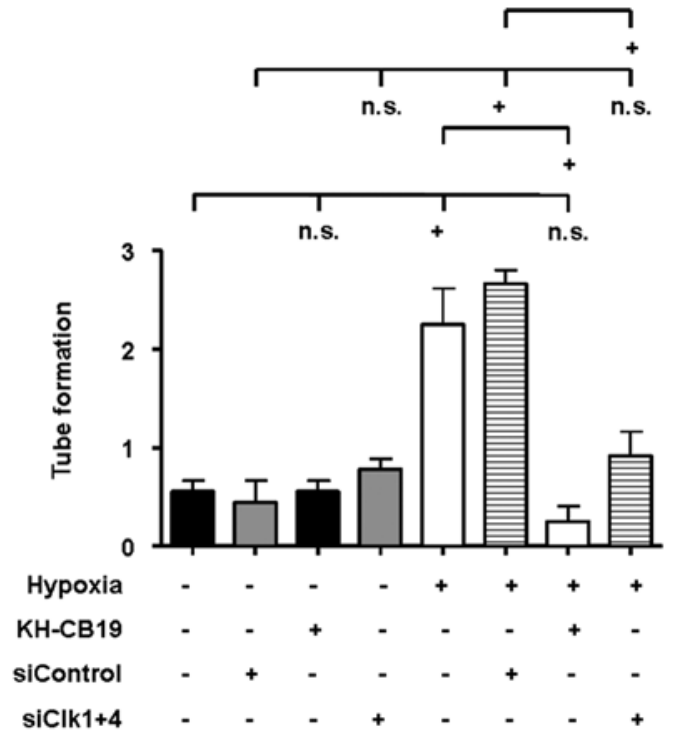

Figure 7. Impact of $\mathrm{Clk} 1$ and 4 inhibition on the pro-angiogenic potential of A549 cells. (A) Depicted is the $\mathrm{x}$-fold induction of tube formation by HMEC-1 cells treated with the supernatant of A549 lung cancer cells. A549 cell were pre-treated for $1 \mathrm{~h}$ with the pharmacologic $\mathrm{Clk}$ inhibitor $(\mathrm{KH}-$ $\mathrm{CB} 19 ; 10 \mu \mathrm{M}$ ), or transfected with 100 pmol of specific siRNAs against Clk1 and $\mathrm{Clk} 4(\mathrm{siClk} 1+4)$ for $48 \mathrm{~h}$ before incubation under hypoxic conditions for $24 \mathrm{~h}$. A scrambled, nonsense siRNA (siControl) was used as control. The proangiogenic potential of A549 cells treated under normoxia versus hypoxia, respectively, was evaluated. The means \pm SEM are shown. ${ }^{+} \mathrm{P}<0.01$; n.s., no significant difference; $\mathrm{n} \geq 3$.

\section{Discussion}

Our results show that hypoxia induces the expression of the TF isoforms as well as of alternative splicing factors and the pro-angiogenic factor Cyr61. Moreover, we found that asTF overexpression in human A549 lung cancer cells increased the proliferative and pro-angiogenic properties of these cells without affecting cellular thrombogenicity. Our data further suggest that these effects are, at least in part, mediated by the induction of the pro-migratory and pro-angiogenic factors Cyr61, MCP-1 and VEGF, as well as integrin $\alpha_{v} \beta_{3}$. Furthermore, we showed for the first time that TF isoform expression is modulated at the post-transcriptional level via Clk-mediated splicing processes under hypoxic conditions and that this modulation can directly alter the pro-angiogenic potential of A549 cells.

Hypoxia induces the pro-angiogenic potential. The transcription factor HIF-1 $\alpha$ plays an essential role in the hypoxic response in cancer as well as in other cells and tissues (24). As reported in the literature, hypoxia induces the expression of HIF-1 $\alpha$ (25). Consistent with this, we also found hypoxia to induce the expression of HIF- $1 \alpha$ in our experiments indicating an adequate hypoxic response of A549 cells in our experimental setting. Moreover, we demonstrated that hypoxic conditions $\left(\mathrm{O}_{2}\right.$ concentration 3\%) induces the expression of alternative splicing-regulating factors, such as Clk1 and Clk4 as well as the phosphorylation of splicing factors including SRp75. This is in line with previous results showing that hypoxia can modulate alternative splicing and expression of alternatively spliced isoforms $(26,27)$. The regulation of alternative splicing plays an essential role in several types of cancer cells as well as in the pathophysiology of malignant diseases $(27,28)$. In our study, we found hypoxia to induce the expression of both TF isoforms asTF and flTF. In line with previous data, total asTF mRNA levels are generally lower than that of fiTF $(5,13)$. Furthermore, hypoxia induced the expression of the proangiogenic factor Cyr61 in our A549 model system. This was associated with an increased pro-angiogenic potential of the cells. Cyr61 and fTF are known to modulate angiogenesis in cancer $(17,21)$. AsTF was shown to be a pro-angiogenic factor in non-cancer settings, such as endothelial cells or cardiomyocytes $(10,11,13)$. However, the role of asTF in cancer-associated angiogenesis is largely unknown. Assuming that asTF also mediates pro-angiogenic effects under hypoxic conditions in lung cancer cells, we evaluated the impact of asTF on the proangiogenic potential of A549 lung cancer cells.

Effects of asTF overexpression on angiogenesis and cell number. Data obtained by Hobbs et al (12) indicated that asTF may play a role in tumor growth and tumor-associated angiogenesis in vitro and in vivo. Those experiments demonstrated that asTF overexpression in pancreatic cancer cells increases tumor cell proliferation. In line with these data, stable asTF overexpression also led to an increased cell number in human A549 lung cancer cells in our study. Moreover, we found asTF overexpression to be associated with higher expression levels of the pro-angiogenic and proliferation-promoting factors Cyr61 (17,20), MCP-1 $(15,29)$ and $\operatorname{VEGF}(16,30)$. In murine cardiomyocytic cells, asTF was also shown to induce murine VEGF and Cyr61 (10). Here, we showed that antibody-mediated inhibition of these factors reduced both the asTF-mediated increase in cell number as well as the proangiogenic effect of asTF overexpression in A549 cells. This is in line with a recently published study of Arderiu et al (31) who demonstrated that TF regulates the generation of MCP-1 in endothelial cells which in turn mediates the pro-angiogenic effect by recruitment of smooth muscle cells to endothelial cells. TF isoforms were also shown to induce the expression of the pro-angiogenic factors VEGF and Cyr61 $(10,32)$. Moreover, A549 cells were found to express VEGF receptors (33). Furthermore, VEGF treatment increased A549 cell survival (33). Therefore, it is possible that asTF mediates its effects on cell number and tube formation via the induction of pro-angiogenic and proliferation-promoting factors, such as MCP-1, VEGF and Cyr61.

In 2009, van den Berg et al (13) showed asTF to induce angiogenesis in endothelial cells. The authors found blockade of $\beta_{1}$ and $\beta_{3}$ integrins as well as the treatment with a TF antibody inhibits the pro-angiogenic effect of asTF. It was suggested that the pro-angiogenic effects of asTF may be mediated directly via asTF-integrin signaling (13). In our experiments, pharmacologic inhibition of $\alpha_{v} \beta_{3}$ integrins by cRGD peptides also reduced the cell number of A549 cells as well as the pro-angiogenic effect on endothelial tube formation. Consistent with this, Loges et al (34) demonstrated that pharmacologic inhibition of integrin $\alpha_{v} \beta_{3}$ by cRGD peptides (cilengitide) reduces the proliferation of human endothelial progenitor cells in vitro. Notably, $\alpha_{v} \beta_{3}$ was reported to be an important mediator of Cyr61 and VEGF functions $(18,19)$. 
Total TF was found to trigger the proliferation of human vascular cells (35). Yet, no asTF-specific inhibitory siRNA or pharmacologic inhibitor has been reported. It is noteworthy that neutralization of flTF had no influence on the asTF-mediated increase in the cell number of A549 cells or endothelial cell tube formation in our experiments. This may be due to the fact that overexpression of the soluble TF isoform had no impact on flTF expression in A549 cells (Fig. 2). Therefore, the asTF-induced increase in cell number and the pro-angiogenic effect were independent of fITF and probably not mediated via flTF upregulation. Based on our results, we hypothesize that the increase in cell number as well as the pro-angiogenic effect of asTF overexpression in A549 lung carcinoma cells is, at least in part, directly mediated by asTF-integrin $\alpha_{v} \beta_{3}$ signaling or indirectly via an asTF-induced increase in the expression of the proliferation-promoting factors Cyr61, MCP-1 and VEGF, which may also be associated with integrin $\alpha_{v} \beta_{3}$ signaling.

Role of Clk-regulated alternative splicing under hypoxic conditions. Alternative splicing is essential for the regulation of the functional diversity and the plasticity of the proteome at the post-transcriptional level in response to environmental changes $(11,28)$. Serine/arginine-rich $(\mathrm{SR})$ protein kinases, such as the Cdc2-like kinases, DNA topoisomerase I and protein kinase $\mathrm{B}$ are known to control alternative splicing processes via modulation of SR protein phosphorylation (7,36-38). Hypoxia was also shown to modulate alternative splicing in normal tissues as well as in cancer cells $(9,27)$. In line with this, we found that hypoxic conditions induce the expression of $\mathrm{Clk} 1$ and Clk4, as well as the phosphorylation of SR proteins, such as SRp75. Moreover, we found that a low oxygen environment promotes the expression of both flTF as well as of the alternatively spliced TF isoform. This implies that TF isoform expression may be modulated at the post-transcriptional level by alternative splicing. In this context, we investigated the role of Clks in hypoxia-induced TF isoform expression. We found that both pharmacologic suppression using specific inhibitors as well as siRNA-mediated gene-silencing of $\mathrm{Clk} 1$ and $\mathrm{Clk} 4$ reduced the flTF and asTF expression in A549 cells under hypoxic as well as normoxic conditions. In line with this, we and others have previously shown that Clks modulate TF isoform expression in TNF- $\alpha$-stimulated human endothelial cells and in thrombin-treated platelets $(4,23)$. In addition, Schwertz et al (23) demonstrated that the thrombin-induced activation of platelets results in post-transcriptional processing of the TF pre-mRNA ( $\mathrm{pTF}$ ) to the spliced mature form of the TF mRNA (mTF). It was also shown that thrombin-induced splicing led to a reduced level of the pTF form and an increased amount of the mature mTF form in platelets. Moreover, pharmacologic inhibition of Clks prevented thrombin-induced processing of the TF pre-mRNA in activated platelets (23). Consistent with these data, we found hypoxia to induce processing of the unspliced TF pre-mRNA (pTF) to the spliced mature mTF form as assessed by RT-PCR, indicated by reduced levels of the pTF form and an increase in mTF mRNA under hypoxic conditions compared to normoxic conditions. Inhibition of Clk1 and Clk4 by KH-CB19 completely abolished this hypoxia-induced effect on TF splicing, which is also in line with the data of Schwertz et al (23). Under normoxic conditions, inhibition of $\mathrm{Clk} 1$ and $\mathrm{Clk} 4$ also led to an increased amount of pTF and reduced mTF mRNA compared to control cells incubated under normoxic conditions. This may be due to the fact that A549 cells also express TF under basal conditions (Fig. 6). Therefore, TF pre-mRNA can also be processed under normal conditions. Since Clks are involved in alternative as well as in constitutive splicing $(23,39)$, inhibition of Clks may also affect mRNA splicing under basal normoxic conditions in A549 cells supporting the hypothesis that Clks modulate splicing of the TF pre-mRNA under both normoxic as well as hypoxic conditions.

Together, our data demonstrated that asTF is a proangiogenic factor and increases cell proliferation in A549 cells. We identified $\mathrm{Clk} 1$ and $\mathrm{Clk} 4$ as modulators of TF isoform expression affecting both splicing of TF pre-mRNA and protein expression of the TF isoforms under hypoxic as well as normoxic conditions in A549 lung carcinoma cells. Furthermore, inhibition of Clk1 and Clk4 was found to influence the pro-angiogenic potential of A549 cells under hypoxic conditions.

Therefore, we conclude that the TF isoform expression is modulated by Clk-mediated alternative splicing at the post-transcriptional level in A549 lung carcinoma cells under hypoxia as well as under normoxia. This in turn controls the pro-angiogenic potential of these cells, which is, at least in part, mediated via the asTF-mediated induction of the downstream effectors Cyr61, MCP-1 and VEGF.

\section{Acknowledgements}

This study was supported by grants from the Deutsche Forschungsgemeinschaft (DFG) (SFB-TR19 to U.R. and H.-P.S.), and the Else Kröner-Fresenius-Stiftung (P09/08// A129/07 to U.R.).

\section{References}

1. Eisenreich A, Celebi O, Goldin-Lang P, Schultheiss HP and Rauch U: Upregulation of tissue factor expression and thrombogenic activity in human aortic smooth muscle cells by irradiation, rapamycin and paclitaxel. Int Immunopharmacol 8: 307-311, 2008.

2. Giesen PL, Rauch U, Bohrman B, et al: Blood-borne tissue factor: another view of thrombosis. Proc Natl Acad Sci USA 96: 2311-2315, 1999.

3. Rauch U, Antoniak S, Boots M, et al: Association of tissuefactor upregulation in squamous-cell carcinoma of the lung with increased tissue factor in circulating blood. Lancet Oncol 6: 254, 2005.

4. Szotowski B, Antoniak S, Poller W, Schultheiss HP and Rauch U: Procoagulant soluble tissue factor is released from endothelial cells in response to inflammatory cytokines. Circ Res 96: 1233-1239, 2005

5. Bogdanov VY, Balasubramanian V, Hathcock J, Vele O, Lieb M and Nemerson Y: Alternatively spliced human tissue factor: a circulating, soluble, thrombogenic protein. Nat Med 9: 458-462, 2003.

6. Eisenreich A, Bogdanov VY, Zakrzewicz A, et al: Cdc2-like kinases and DNA topoisomerase I regulate alternative splicing of tissue factor in human endothelial cells. Circ Res 104: 589-599, 2009.

7. Eisenreich A, Malz R, Pepke W, Ayral Y, Poller W, Schultheiss HP and Rauch U: Role of the phosphatidylinositol 3-kinase/protein kinase $B$ pathway in regulating alternative splicing of tissue factor mRNA in human endothelial cells. Circ J 73: 1746-1752, 2009.

8. Tardos JG, Eisenreich A, Deikus G, et al: SR proteins ASF/SF2 and SRp55 participate in tissue factor biosynthesis in human monocytic cells. J Thromb Haemost 6: 877-884, 2008. 
9. Rauch U and Antoniak S: Tissue factor-positive microparticles in blood associated with coagulopathy in cancer. Thromb Haemost 97: 9-10, 2007.

10. Eisenreich A, Boltzen U, Malz R, Schultheiss HP and Rauch U: Overexpression of alternatively spliced tissue factor induces the pro-angiogenic properties of murine cardiomyocytic HL-1 cells. Circ J 75: 1235-1242, 2011

11. Eisenreich A and Rauch U: Regulation and differential role of the tissue factor isoforms in cardiovascular biology. Trends Cardiovasc Med 20: 199-203, 2010.

12. Hobbs JE, Zakarija A, Cundiff DL, et al: Alternatively spliced human tissue factor promotes tumor growth and angiogenesis in a pancreatic cancer tumor model. Thromb Res 120 (Suppl 2) S13-S21, 2007

13. van den Berg YW, van den Hengel LG, Myers HR, et al: Alternatively spliced tissue factor induces angiogenesis through integrin ligation. Proc Natl Acad Sci USA 106: 19497-19502, 2009.

14. Goldin-Lang P, Tran QV, Fichtner I, et al: Tissue factor expression pattern in human non-small cell lung cancer tissues indicate increased blood thrombogenicity and tumor metastasis. Oncol Rep 20: 123-128, 2008.

15. Zhang T, Koide N, Wada Y, et al: Significance of monocyte chemotactic protein-1 and thymidine phosphorylase in angiogenesis of human cardiac myxoma. Circ J 67: 54-60, 2003.

16. Zhang Y, Han J, Yang X, et al: Pigment epithelium-derived factor inhibits angiogenesis and growth of gastric carcinoma by downregulation of VEGF. Oncol Rep 26: 681-686, 2011.

17. Shimizu T, Okayama A, Inoue T and Takeda K: Analysis of gene expression during staurosporine-induced neuronal differentiation of human prostate cancer cells. Oncol Rep 14: 441-448, 2005.

18. Chen N, Leu SJ, Todorovic V, Lam SC and Lau LF: Identification of a novel integrin $\alpha_{\mathrm{v}} \beta_{3}$ binding site in CCN1 (CYR61) critical for pro-angiogenic activities in vascular endothelial cells. J Biol Chem 279: 44166-44176, 2004.

19. Hutchings $H$, Ortega $N$ and Plouët J: Extracellular matrix-bound vascular endothelial growth factor promotes endothelial cell adhesion, migration, and survival through integrin ligation. FASEB J 17: 1520-1522, 2003.

20. Löbel M, Bauer S, Meisel C, et al: CCN1: CCN1: a novel inflammation-regulated biphasic immune cell migration modulator. Cell Mol Life Sci 69: 3101-3113, 2012.

21. Schaffner F, Versteeg HH, Schillert A, Yokota N, Petersen LC, Mueller BM and Ruf W: Cooperation of tissue factor cytoplasmic domain and PAR2 signaling in breast cancer development. Blood 116: 6106-6113, 2010.

22. Fedorov O, Huber K, Eisenreich A, et al: Specific CLK inhibitors from a novel chemotype for regulation of alternative splicing. Chem Biol 18: 67-76, 2011.

23. Schwertz H, Tolley ND, Foulks JM, et al: Signal-dependent splicing of tissue factor pre-mRNA modulates the thrombogenicity of human platelets. J Exp Med 203: 2433-2440, 2006.

24. Kim SH, Kim KW and Jeong JW: Inhibition of hypoxia-induced angiogenesis by sodium butyrate, a histone deacetylase inhibitor, through hypoxia-inducible factor-1 $\alpha$ suppression. Oncol Rep 17: 793-797, 2007
15. Łuczak MW, Roszak A, Pawlik P, Kedzia H, Lianeri M and Jagodziński PP: Increased expression of HIF-1A and its implication in the hypoxia pathway in primary advanced uterine cervical carcinoma. Oncol Rep 26: 1259-1264, 2011.

26. Gang H, Hai Y, Dhingra R, et al: A novel hypoxia-inducible spliced variant of mitochondrial death gene Bnip3 promotes survival of ventricular myocytes. Circ Res 108: 1084-1092, 2011.

27. Hirschfeld M, zur Hausen A, Bettendorf H, Jäger $M$ and Stickeler E: Alternative splicing of Cyr61 is regulated by hypoxia and significantly changed in breast cancer. Cancer Res 69: 2082-2090, 2009.

28. Tazi J, Bakkour N and Stamm S: Alternative splicing and disease. Biochim Biophys Acta 1792: 14-26, 2009.

29. Xu F, Shi J, Yu B, Ni W, Wu X and Gu Z: Chemokines mediate mesenchymal stem cell migration toward gliomas in vitro. Oncol Rep 23: 1561-1567, 2010.

30. Zhao X, Li DC, Zhao H, et al: A study of the suppressive effect on human pancreatic adenocarcinoma cell proliferation and angiogenesis by stable plasmid-based siRNA silencing of c-Src gene expression. Oncol Rep 27: 628-636, 2012.

31. Arderiu G, Peña E, Aledo R, Juan-Babot O and Badimon L: Tissue factor regulates microvessel formation and stabilization by induction of chemokine (C-C motif) ligand 2 expression. Arterioscler Thromb Vasc Biol 31: 2607-2615, 2011.

32. Ollivier V, Bentolila S, Chabbat J, Hakim J and de Prost D: Tissue factor-dependent vascular endothelial growth factor production by human fibroblasts in response to activated factor VII. Blood 91: 2698-2703, 1998

33. Roberts JR, Perkins GD, Fujisawa T, Pettigrew KA, Gao F, Ahmed A and Trickett DR: Vascular endothelial growth factor promotes physical wound repair and is anti-apoptotic in primary distal lung epithelial and A549 cells. Crit Care Med 35: 2164-2170, 2007

34. Loges S, Butzal M, Otten J, et al: Cilengitide inhibits proliferation and differentiation of human endothelial progenitor cells in vitro. Biochem Biophys Res Commun 357: 1016-1020, 2007.

35. Cirillo $\mathrm{P}$, Calì G, Golino $\mathrm{P}$, et al: Tissue factor binding of activated factor VII triggers smooth muscle cell proliferation via extracellular signal-regulated kinase activation. Circulation 109: 2911-2916, 2004.

36. Bourgeois CF, Lejeune F and Stévenin J: Broad specificity of SR (serine/arginine) proteins in the regulation of alternative splicing of pre-messenger RNA. Prog Nucleic Acids Res Mol Biol 78: 37-88, 2004.

37. Eisenreich A, Boltzen U, Poller W, Schultheiss HP and Rauch U: Effects of the Cdc2-like kinase-family and DNA topoisomerase I on the alternative splicing of eNOS in TNF- $\alpha$-stimulated human endothelial cells. Biol Chem 389: 1333-1338, 2008.

38. Eisenreich A and Rauch U: PI3K inhibitors in cardiovascular disease. Cardiovasc Ther 29: 29-36, 2011.

39. Prasad J, Colwill K, Pawson T and Manley JL: The protein kinase Clk/Sty directly modulates SR protein activity: both hyper- and hypophosphorylation inhibit splicing. Mol Cell Biol 19: 6991-7000, 1999. 